\section{Cursos masivos abiertos en línea de psicoterapia}

\section{Massive open online courses on psychotherapy}

\section{Sr. Editor}

Los desórdenes mentales son un problema prioritario de salud pública, de manera que su prevalencia mundial se calcula en $17,6 \%{ }^{1}$, y se estima que el $22,9 \%$ de años vividos con discapacidad a nivel mundial se deben a desórdenes mentales y abuso de sustancias ${ }^{2}$.

Para afrontar esta situación, resulta necesario que los sistemas de salud dispongan de personal capacitado en el diagnóstico y manejo de estas condiciones, que incluye el manejo de psicoterapiabásica. De esta manera, un metaanálisis refiere que el entrenamiento en psicoterapia del personal de salud no especializado es efectivo en el primer nivel de atención, reduciendo los desórdenes mentales perinatales en mujeres ${ }^{3}$ y el riesgo de padecer depresión en adultos hasta en $60 \%$, siendo potencialmente efectivo para otros desórdenes mentales ${ }^{4}$. Coherentemente, la normativa peruana refiere que los encargados de realizar el diagnóstico y manejo en el primer nivel de atención son los médicos y todo personal asistencial que brinde servicio de salud,previamente capacitado ${ }^{5}$. Sin embargo, lograr esta capacitación es difícil, en especial en países con escasos recursos destinados a salud.

Ante la necesidad de realizar capacitaciones masivas al personal de salud, surgen como iniciativas de gran potencial los cursos masivos abiertos en línea (MOOC, por sus siglas en inglés), los cuales emplean recursos audiovisuales (videoclases, audios, foros) con la finalidad de capacitar masivamente a los interesados en cierto tema. Con el objetivo de evaluar los MOOC disponibles sobre psicoterapia, realizamos una búsqueda en las plataformas web más populares de MOOC (COURSERA, EDX, Future Learning, CANVAS Network, UniMOOC, MiríadaX, entre otros), así como en el buscador Google. 
Tabla 1. MOOC de Psicoterapia encontrados $(n=5)$

\begin{tabular}{|c|c|c|c|c|c|c|c|c|}
\hline Plataforma & $\begin{array}{l}\text { Título del } \\
\text { curso }\end{array}$ & Institución & $\begin{array}{c}\text { Idioma/ } \\
\text { ¿Subtítulos } \\
\text { en español? }\end{array}$ & $\begin{array}{l}\text { ¿Posee } \\
\text { video- } \\
\text { clases? }\end{array}$ & $\begin{array}{c}\text { Precio/ } \\
\text { Precio del } \\
\text { certificado }\end{array}$ & $\begin{array}{c}\text { Brinda } \\
\text { certificado }\end{array}$ & $\begin{array}{l}\text { Incluye } \\
\text { manejo de } \\
\text { depresión }\end{array}$ & $\begin{array}{c}\text { Incluye } \\
\text { manejo de } \\
\text { alcoholismo }\end{array}$ \\
\hline COURSERA & $\begin{array}{l}\text { Primero Auxi- } \\
\text { lios Psicológi- } \\
\text { cos (PAP) }\end{array}$ & $\begin{array}{l}\text { Universidad } \\
\text { Autónoma } \\
\text { de Barcelona, } \\
\text { España }\end{array}$ & Español/No & Sí & No/29 USD & No & No & No \\
\hline COURSERA & $\begin{array}{l}\text { Psychological } \\
\text { First Aid }\end{array}$ & $\begin{array}{l}\text { Johns Hopkins } \\
\text { University, } \\
\text { EE.UU. }\end{array}$ & Inglés/No & Sí & No/49 USD & Sí & No & No \\
\hline FUTURELEARN & $\begin{array}{l}\text { Psychology } \\
\text { and Mental } \\
\text { Health: } \\
\text { Beyond Nature } \\
\text { and Nurture }\end{array}$ & $\begin{array}{l}\text { University } \\
\text { of Liverpool, } \\
\text { Inglaterra }\end{array}$ & Inglés/No & Sí & $\mathrm{No} / 49 \mathrm{f}$ & Sí & No & No \\
\hline IASE & $\begin{array}{l}\text { Iniciación a la } \\
\text { terapia familia }\end{array}$ & IASE, España & Español/No & Sí & No/No & Sí & No & No \\
\hline IAEU & $\begin{array}{l}\text { Intervenciones } \\
\text { breves para } \\
\text { reducir el } \\
\text { consumo de } \\
\text { alcohol }\end{array}$ & $\begin{array}{l}\text { Universidad } \\
\text { Nacional de } \\
\text { Mar del Plata, } \\
\text { Argentina }\end{array}$ & Español/No & No & $\mathrm{No} / 25 \square$ & Sí & No & Sí \\
\hline
\end{tabular}

Utilizamos los términos clave: [psicoterapia, psicoterapia breve, psicoterapia racional emotiva, psicoterapia psicodinámica, psicoterapia grupal, terapia cognitiva, terapia conductual, terapia gestáltica, terapia psicoanalítica, terapia socioambiental], así como sus traducciones al inglés. Elegimos aquellos MOOC que tengan entre sus objetivos principales enseñar algún tipo de psicoterapia al personal de salud.

Encontramos cinco MOOC disponibles que se dedican a educar en la realización de alguna terapia psicológica;tres fueron respaldados por universidades de Europa y uno por una universidad de Estados Unidos. Cuatro cursos emplearon videoclases, mientras que el restante utilizó únicamente lecturas. Tres cursos presentan su contenido en español, solo un curso incluye en su temario el manejo del alcoholismo y ninguno abarca el manejo de trastornos depresivos. Todos los cursos son brindados gratuitamente, sin embargo, para obtener la certificación en cuatro de ellos se debe pagar cincuenta dólares en promedio (Tabla 1).

Como se observa, son pocos los MOOC disponibles para este fin que pueden ser utilizados por los sistemas de salud de Latinoamérica para la capacitación de sus trabajadores, por lo cual se estaría desaprovechando esta alternativa de capacitación masiva. Por ello, hacemos un llamado a las instituciones pertinentes, para que apoyen la creación de MOOC sobre psicoterapias en castellano, que incluyala prevención y el manejo de las condiciones más frecuentes (depresión y alcoholismo).
Asimismo, a los sistemas de salud para que hagan uso de este material con el fin de mejorar la formación del personal sanitario del primer nivel.

Diego Urrunaga-Pastor ${ }^{1}$, Angie Mariños-Claudet ${ }^{1}$, Julio Cjuno ${ }^{2,4}$, C. Mahony Reátegui-Rivera ${ }^{3}$, Alvaro Taype-Rondan ${ }^{4}$

${ }^{1}$ Sociedad Científica de Estudiantes de Medicina de la Universidad de San Martín de Porres, Facultad de Medicina Humana, Universidad de San Martín de Porres Lima, Perú.

${ }^{2}$ Instituto de Investigación de la Universidad Católica los Ángeles de Chimbote, Chimbote, Áncash, Perú. ${ }^{3}$ Asociación para el Desarrollo de la Investigación Estudiantil en ciencias de la Salud (ADIECS), Lima, Perú. ${ }^{4}$ CRONICAS Centro de Excelencia en Enfermedades Crónicas, Universidad Peruana Cayetano Heredia,

Lima, Perú.

\section{Referencias}

1. Steel Z, Marnane C, Iranpour C, Chey T, Jackson JW, Patel $\mathrm{V}$, et al. The global prevalence of common mental disorders: a systematic review and meta-analysis 1980-2013. International Journal of Epidemiology 2014; dyu038.

2. Whiteford HA, Degenhardt L, Rehm J, Baxter AJ, Ferrari AJ, Erskine HE, et al. Global burden of disease attributable to mental and substance use disorders: findings from the Glo- 
bal Burden of Disease Study 2010. Lancet 2013; 382 (9904): 1575-86.

3. Clarke K, King M, Prost A. Psychosocial interventions for perinatal common mental disorders delivered by providers who are not mental health specialists in low-and middle-income countries: a systematic review and meta-analysis. PLoS Med 2013; 10 (10): e1001541.

4. Van Ginneken N, Tharyan P, Lewin S, Rao GN, Meera S, Pian J, et al. Non-specialist health worker interventions for the care of mental, neurological and substance-abuse disorders in low-and middle-income countries. The Cochrane Library. 2013.

5. Ley General de Salud. Ley No 26842. Diario Oficial El Peruano (20 de julio de 1997). 2006.
Financiamiento: El presente estudio ha sido autofinanciado. Conflictos de interés: Los autores declaran no tener conflictos de interés con respecto a este artículo.

Correspondencia:

Diego Alonso Urrunaga Pastor

Av. Bolognesi 450, La Punta, Callao, Perú.

diego_urrunaga@usmp.pe

Angie Mariños-Claudet angie_mclaudet@hotmail.com

Julio Cjuno

juliocjunosuni@gmail.com

C. Mahony Reátegui-Rivera mahony3093@gmail.com

Alvaro Taype-Rondan alvaro.taype.r@upch.pe 